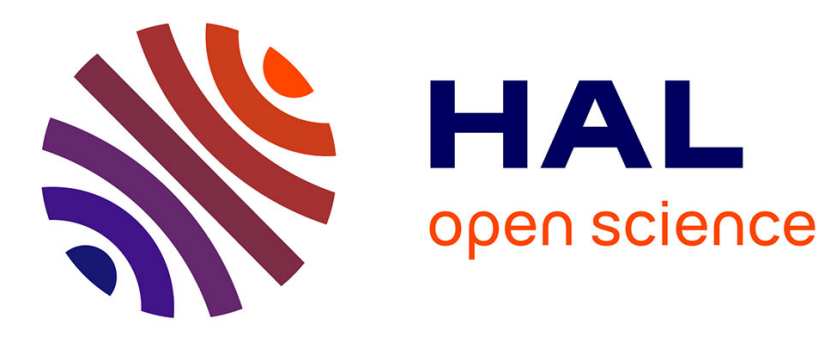

\title{
QoE aware Service delivery in distributed environment
}

Khalil Ur Rehman Laghari, Noel Crespi, Benjamin Molina, Carlos E. Palau

\section{To cite this version:}

Khalil Ur Rehman Laghari, Noel Crespi, Benjamin Molina, Carlos E. Palau. QoE aware Service delivery in distributed environment. WAINA 2011: IEEE Workshops of International Conference on Advanced Information Networking and Applications, Mar 2011, Singapour, Singapore. pp.837-842, 10.1109/WAINA.2011.58 . hal-01303062

\section{HAL Id: hal-01303062 \\ https://hal.science/hal-01303062}

Submitted on 15 Apr 2016

HAL is a multi-disciplinary open access archive for the deposit and dissemination of scientific research documents, whether they are published or not. The documents may come from teaching and research institutions in France or abroad, or from public or private research centers.
L'archive ouverte pluridisciplinaire HAL, est destinée au dépôt et à la diffusion de documents scientifiques de niveau recherche, publiés ou non, émanant des établissements d'enseignement et de recherche français ou étrangers, des laboratoires publics ou privés. 


\section{QoE aware Service Delivery in Distributed Environment}

\author{
Khalil ur Rehman Laghari,Noel Crespi \\ Networks and Mobile Multimedia Service \\ Institut Telecom, Telecom SudParis \\ Evry, France \\ khalil.laghari@it-sudparis.eu,noel.crespi@it- \\ sudparis.eu
}

\author{
B. Molina, C.E. Palau \\ Communications Department \\ Universidad Politécnica de Valencia \\ Valencia, Spain \\ benmomo@upvnet.upv.es, cpalau@dcom.upv.es
}

\begin{abstract}
Service delivery and customer satisfaction are strongly related items for a correct commercial management platform. Technical aspects targeting this issue relate to QoS parameters that can be handled by the platform, at least partially. Subjective psychological issues and human cognitive aspects are typically unconsidered aspects and they directly determine the Quality of Experience (QoE). These factors finally have to be considered as key input for a successful business operation between a customer and a company. In our work, a multi-disciplinary approach is taken to propose a QoE interaction model based on the theoretical results from various fields including pyschology, cognitive sciences, sociology, service ecosystem and information technology. In this paper a QoE evaluator is described for assessing the service delivery in a distributed and integrated environment on per user and per service basis.
\end{abstract}

Keywords: Quality of Experience, Psychological Models, Quality of Service, Distributed Environment, Business Model, Service Delivery.

\section{INTRODUCTION}

Nowadays, networking and telecommunication infrastructures have gained a mature status where end users feel confident enough to apply for more customized ones. Different architectures (user-centric, service-centric) have been proposed to design and deploy enhanced systems to provide rich service usage experience. Here the term experience is a key differentiation factor that encompasses multiple dimensions of the interaction between customer and company in business applications. In some transactions (e.g. payment actions) security is a fundamental issue: confidentiality is typically provided by HTTPS in the Internet, whereas authenticity relates more to digital signatures. In other environments (e.g. multimedia applications) resource allocation is mandatory in order to guarantee specific quality in the offered service: IP DiffServ [1] provides a reasonable (coarse-grained) performance for moderate network traffic whereas IP Intserv [1] can fulfill the demanded (fine-grained) requirements in congested environments for any service type. However resource allocation only maps to concrete network parameters such as bandwidth, delay, packet loss and jitter. Such parameters can be easily measured at network level and often refer to (network) Quality of Service (QoS) parameters. For each application or service type, specific QoS parameters have to be assigned in order to provide a good service, e.g. a video application will require more bandwidth than a voice application, but the delay and jitter in the second application might be more stringent. Traditionally, QoS (Quality of Service) parameters are considered as bench mark for service quality and customer satisfaction in conventional service solutions. QoS is more oriented towards networks and applications; hence QoS parameters are unable to satisfy the aesthetic and hedonic needs of users and customers.

We live in the new era of customization and pervasive computing. This means that all customers want something special related to them whenever a requested service is being delivered to them. And the way the user interacts with the telecommunication infrastructure should be transparent. In order to tackle these issues, some of following efforts were taken.

A service profile [2] was an effort towards a better user experience. It follows user's preferences and configuration values resulting in a customized service delivery. Whenever a user first enters a specific system or service, it has to create a profile. This normally implies filling an endless document in form of web forms. Such situation is not comfortable for the user as it has to enter many pieces of information, and sometimes it can be really annoying if the same information has to be entered for each service type without being able to import/export data. Automatisation was a need. Some applications such as eBay can automatically build user profiles by means of tracing user's past and current actions. This paper will not focus on concrete user profile information (bottom-up approach), but will consider general features from users to profile them into different categories (top-down approach).

Context enablers [3] have been proposed in the last years in order to automatically retrieve user information and decide in real time the best service delivery method. Mobile devices such as iPhone and Android enabled phones are able to send GPS coordinates to remote applications. Such enablers will be used in our paper.

The latest trend for improving user experience is Quality of Experience (QoE), which represents the human centric quality aspects, unlike QoS which is merely a technology centric approach. QoE is a blue print of all human needs, desires and perceptions concerning a service and/or product in a specific context. Higher QoE promises happier user 
experience as well as surge in customer loyalty while lower QoE brings poor user/customer experience and increases the customer churn rate. In distributed environment, services, contents, devices and resources may not only be spread in various locations but they are of heterogeneous nature too (e.g., Web and Telco environment). In such an environment, ensuring QoE aware service delivery is a challenging task primarily because QoE factors are really difficult to identify, obtain, and measure. Matching QoE factors with service features, business elements and service performance parameters of a system is one of the main challenges. Moreover, technicians and psychologists need to work together in order to propose useful models that bring value to service delivery and business. The paper investigates how to introduce QoE notion for service discovery and delivery in a distributed environment. This implies that when a user looks for a service in a service catalogue, the backend system will provide a list of matching services according to a QoE criterion (matching services are filtered and ranked not only according to QoS parameters but also according to QoE parameters). The same idea applies to service composition.

The paper is structured as follows. Section II describes the theoretical background and discusses how QoE is perceived in current research and what is available in other psychological and cognitive theories which could possibly be used to enrich QoE model. Section III presents our multidisciplinary QoE interaction model. Section IV discusses how this model is realized in order to deliver QoE aware service delivery through a simplified test bed. Section V ends with the conclusions and future work.

\section{THEORETICAL BACKGROUND}

\section{A. QoE as an Extension of $Q o S$}

Primarily the research work in the QoE domain considers QoE as an extension of QoS concept where the end user's perception is mapped to QoS parameters. The work in [5,6 and 7] focuses on this aspect and QoE is evaluated on the basis of users' perception of QoS parameters which are somehow linked to QoE using various mathematical equations. In literature, paper [5] describes a correlation model to evaluate QoE on the basis of QoS parameters at network layer. In [6] authors discuss a logarithmic relationship between QoS parameters and QoE. Authors in [7] describe some QoE to QoS evaluation method based on some differential equations showing the improved performance of an exponential relationship over a logarithmic one. Though an exponential function may provide better results as described in [7] for packet loss ratio, it cannot be generalized for the whole set of QoS parameters; the bandwidth QoS parameter may possibly be linked to QoE through a logarithmic function. Considering the random nature of human behavior and intentions, it is difficult to assure a good accuracy in the (tightly) coupled process of QoS-QoE. We believe that work on QoS is critical, but not sufficient for measuring the total quality of experience. Moreover considering QoS as only representative aspect of QoE will probably devalue other non technical aspects like business factors (cost, promotions, ads, etc) and social aspects and contextual influences upon a person.

\section{B. Pyschological models and Congnitive science}

Mostly related works consider QoE as an extension of QoS concept and they focus more on QoS parameters only neglecting other aspects related to human intentions and behavior, e.g., pre-service human perception, human intentions, role of marketing, social and organizational pressures, business models, context of use, etc. The variation in human behavior is linked to human intentions. Intentions are triggered by some motivational factors which influence human behavior; they are indicators of how hard people are willing to try, how much of an effort they are planning to exert in order to perform the behavior [10]. The use of psychological models and cognitive science could be of great value to understand human intentions and behavior. A widely recognized model is the Technology Acceptance Model (TAM) which is a derivative of the Theory of Reasoned Action (TRA) [8]. TAM is applied to a broad range of information technologies to predict both human intentions and system usage. There are two major predictors of behavioral intention based on the theory of reasoned action:

- Attitude: describes individual's internal positive or negative feelings to perform some behavior or not.

- Subjective Norm/Social Norms (SN): denotes the fundamental social pressures on an individual's perception to perform some behavior or not.

Meanwhile, TAM proposes that perceived ease of use and perceived usefulness of technology are predictors of user attitude towards using the technology [8]. Wanmin $\mathrm{Wu}$ et al. in [17] propose the use of the TAM model as QoE construct in a distributed interactive multimedia environment. In [9] the author uses a TAM model for pervasive computing in order to understand human behavior. However the TAM model severely lacks in addressing the fact that behaviors are often not under volitional control. To target this issue, the Theory of Planned Behavior (TPB) was introduced by I. Ajzen [10]. He proposed an extension to TRA by adding one additional aspect called Perceived Behavioral Control (PBC) in order to reduce the limitations of TRA/TAM. PBC factors refer to whether the person feels in control of the action in question. In our earlier work [4], a simplified customer experience model is proposed based on TPB model and it's transformed into ontological model to accommodate run time service configuration on the basis of customer experience requirements. There is also an extended version of TPB called Decomposed TPB (DTPB) which includes a detailed sub classification of parameters. The DTPB model was found to have better predictive power compared to the traditional Theory of Planned Behavior model and the Technology Acceptance Model [16], further more It provides a comprehensive way to understand how an individual's attitude, subjective norms and perceived behavioral control can influence his or her intention, taking into account: 
- Personal attitude factors: Ease of use, joy of use, usefulness, comfort, complexity, annoyance, boredom, etc.

- Social factors: friend \& family, organizational pressures, legal issues, demographic factors, etc.

- Perceived behavioral control factors: cost, help desk and other facilitating conditions.

The application of psychological models for understanding human intentions and behavior provides a global subjective assessment of user/customer requirements corresponding to a particular service and product. However the use of cognitive science and mental models to understand human task performance can provide precise quantitive information about human performance [11]. In Human Computer Interaction (HCI) and Interface design, some cognitive human performance models are also used to capture human task performance such as GOMS [12]. Therefore it is possible to get quantitative information about humans such as memory capacity, audiovisual level, and reaction time. We will consider objective cognitive information as one of the important QoE factor.

\section{QUALITY OF EXPERIENCE MODEL DESCRIPTION}

QoE is a set of human centric factors which describes all hedonic as well as aesthetic human needs. QoE notion helps us understand human requirements, perceptions and desires concerning a particular product/service/application. The International Telecommunication Union ITU-T defines QoE [13] as "The overall acceptability of an application or service, as perceived subjectively by the end-user". ITU-T vision links QoE with more subjective studies and QoS related work. However we enhance the QoE notion to include objective cognitive factors as well as other non technical factors. As we know the subjective experience of humans can vary from person to person but objective human factors like memory capacity, audiovisual human limits, reaction time and other psychometric aspects can be calculated accurately without conducting extensive user subjective studies. The novelty in our work is to extend the QoE model to include objective human cognitive aspects and incorporate some valid psychological subjective approach for the assessment of QoE. For us, " $Q o E$ is a set of human centric factors based on human subjective aspects and objective cognitive aspects developed in particular context."

Total QoE = Subjective QoE + Objective QoE.

It is important to note that when we say objective QoE, it refers to human cognitive aspects. A generalized $\mathrm{QoE}$ interaction model is presented in Figure 1. This QoE interaction model is founded on a multi-discipliary approach that is based on theoretical results from various fields including pyschology, cognitive science, sociology, service ecosystem and information technology. This model is broadly divided in two parts: QoE domain describes the formation process of Human QoE aspects. Technology \& Business Domain shows simple service ecosystem interaction in distributed environment.

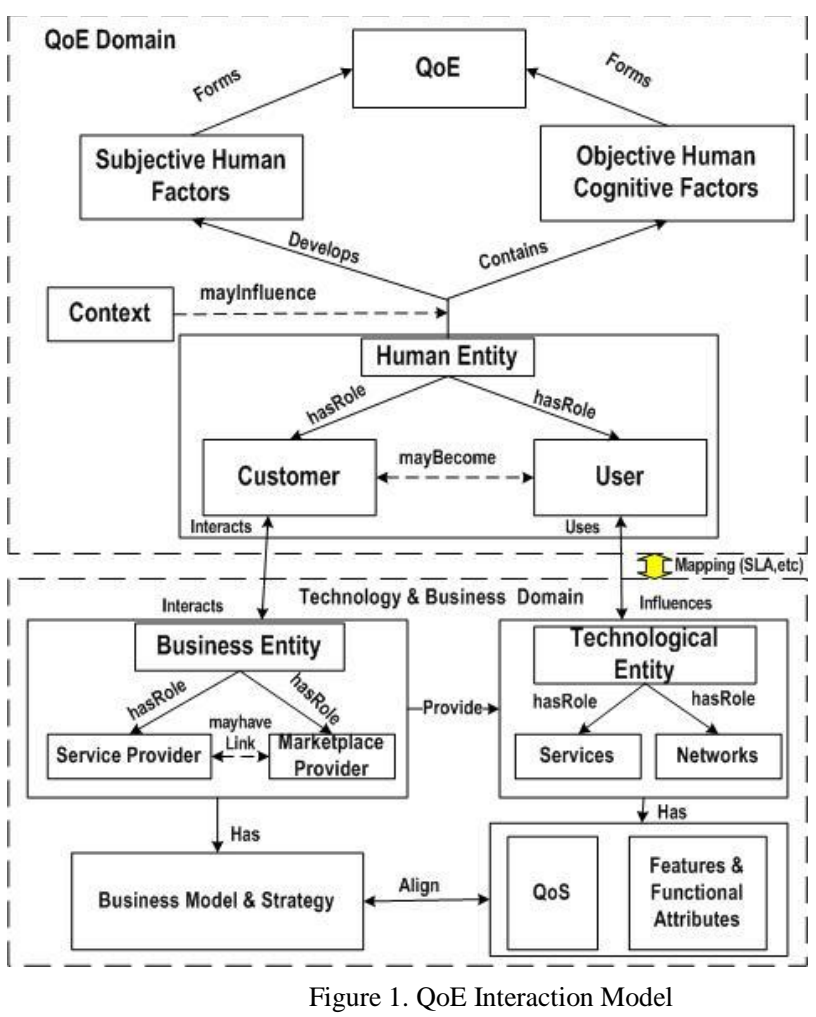

A. QoE domain

Our model is based on a multi-disciplinary approach. In our QoE domain, it is proposed that the human entity forms a set of QoE requirements based on both his/her subjective and objective cognitive factors.

- Subjective human factors are qualitative in nature. They are obtained through surveys, interviews, and opinion polls etc. Some examples of subjective human factors are: usefulness, usability, need, availability, feelings, happiness, boredom, expectations, desires, social influences, brand image, cost, satisfaction, etc. The selection of suitable psychological methodologies depends upon the nature of service and environment. For the subjective assessment of QoE, we use a modified version of the Decomposed Theory of Planned Behavior (DTPB) and the modifications carried out in DTPB are related to classification of DTPB factors and the use of contextual parameters for moderation of DTPB factors.

- Objective human cognitive factors are quantitative factors which predict human performance. Through the use of cognitive science one can obtain the possible set of human reaction time for working memory, as well as visual and auditory human processor aspects. The Human Process Model (HPM) and cognitive psychological models are very effective techniques to understand human cognitive capabilities which use reaction time (RT) as the primary performance measure to infer the possible structure of mental systems [11]. Human reactions 
and emotions change if a system takes longer to respond. For example in web environments, where users are browsing a webpage, they may perceive a system is slow if they don't receive a response within 3 seconds, whereas users lose their attention approximately after 10 seconds in case the system is unable to respond. Moreover, if the waiting time increases beyond 15 seconds, users get completely annoyed. Thus human reaction time is directly related to system reaction time [14]. This piece of information enables a system designer to predict the performance in terms of the amount of time it takes a person to complete a task without performing experiments. Considering its utility, we propose the use of such objective cognitive factor as one important QoE factor.

- The role of the human entity is broadly categorized as user and customer. These roles can be extended to other roles and even they could further be sub classified. Furthermore, we don't consider in our current work any user-to-user interaction as in social media. The customer role is considered as the legal owner of a service whereas the user is the one who actually uses a service. The dotted line between these two roles describes a possibility that a customer may become user or vice versa. This role division also suggests that user experience needs may vary from customer experience needs. Furthermore each role has some characteristics (customer or user profile attributes, age, gender etc) and they may get influenced in some specific context (spatial context such as location, home, office etc, and temporal context such as day, night etc). User or customer characteristics are normally used in psychological assessments for moderation or classification of human experience requirements based on age, profession and gender or in other words profile based classification. However we also propose to moderate QoE factors based on context, because we believe that variation in context (spatial, temporal) may develop different human intentions, feelings and needs, because each role may behave differently in different environments.

\section{B. Technology and Business domain}

In this section, we describe a generalized interaction between various actors of service ecosystem, where Business Entity is shown decoupled from Technological Entity.

- The Business Entity (BE) may have different roles and each role may have some links or contracts for exploiting each others' services and resources. Marketplace Provider, Service Provider (SP), Network Operator (NO), Device Vendor (DV), Content Provider (CP), etc are possible role of BE. Customers establish interaction with them to subscribe to a service that fulfils their intended goals based on some agreed upon Service Level Agreement (SLA). This interaction can be physical or online (direct/indirect) but in both cases this interaction experience also develops positive or negative feelings. The $\mathrm{BE}$ has some specific business model and strategy which defines the direction of its business, and BE may have their own network resources or it may use third party resources. However, there should be an alignment between business and technical entities to create an integrated technical and business solution which could guarantee the QoE requirements.

- The Technological Entity may also have different roles such as service, application, network, device, and resources. Actual service implementations and resources are available included in this entity. Each role has different functional attributes and performance benchmarks. The user can use various technological entities to achieve his goals. The usage experience of these technological entities determines the success and failure of any business. In order to retain customers and provide high quality of experience, it is obligatory that Business and Technology domain aspects completely fulfill the needs and desires of customers and users.

\section{Mapping methodology between domains}

The Mapping between the two domains shows that the QoE domain is influenced by Business and Technology domain aspects and vice versa. The most crucial research challenge is to find an optimal mechanism to map QoE factors into the Technology and Business domain and establish some suitable win-win agreement between two domains. As described in section II, generally conventional QoE approaches describe an analytical approach between QoE and QoS, and they mainly consider QoS as unique QoE construct. In our approach, QoE is a dynamic and diverse set of factors that can influence not only QoS parameters but also business models and service features. Thus we suggest an empirical and adaptive mapping approach rather than using a fixed and analytical mapping approach. The following steps are suggested for mapping:

- Identify and gather QoE subjective and objective factors.

- Analyze the impact of QoE domain data with Technical \& Business domain data based on experiential results and empirical data.

- Establish some flexible and adaptive statistical relationship between them which could facilitate QoE oriented SLA between Business Entity and Customer in distributed environment.

\section{TESTBED}

We would like to test our QoE framework in distributed environment, combining a Telco infrastructure and a Web infrastructure. Such environment is the target of the SERVERY project [15]. SERVERY (advanced SERVice architecture and service delivERY environment) performs research, prototypes and evaluation of an open European Service-marketplace, allowing creation and delivery of multimedia services to Mobile and Fixed Broadband users 
through the combination of the abundant and flexible Internet world with the stable and trustworthy Telco environment.

One basic use case comprises a composite service, where a user wants to get the weather forecast and send it to a friend via SMS (e.g. in order to meet for surfing activities). The former one is a web service, whereas the latter is a Telco service. There is a service repository where the user can issue a (semantic) request for such composite service, and the matching engine is in charge of providing the composed service. In such situation the result should be the best composite service according to QoE parameters, which implies maximizing QoE for each individual service. This means that the service repository holds a list of different 'translation' services, as well as various 'sendSMS' services. Through QoE matching one 'translation' service and one 'sendSMS' service will be selected as optimum and will be combined to provide the composite service.

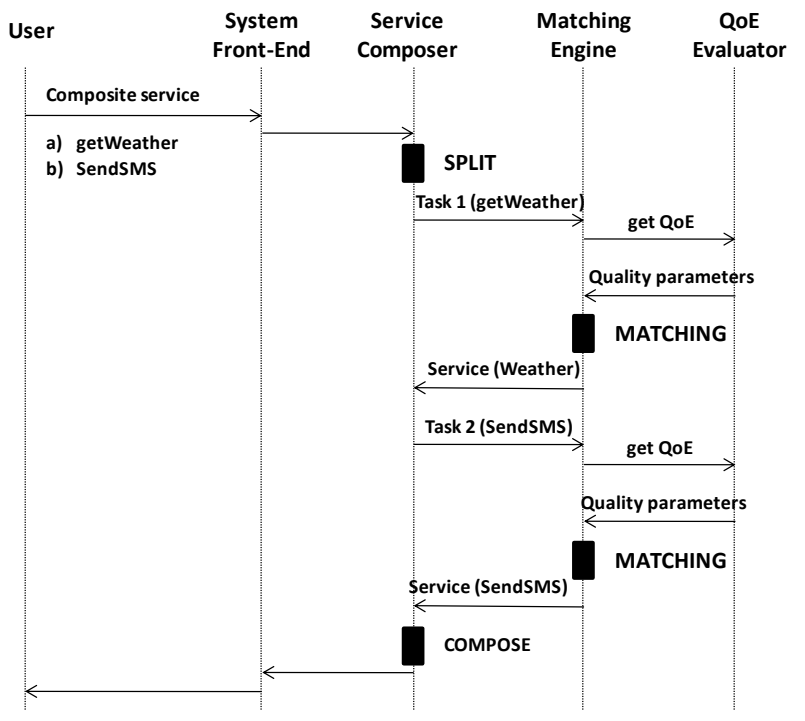

Figure 2. QoE evaluation during discovery process

\section{A. QoE evaluator}

For a composite service, the assessment of QoE takes place at a higher level rather than each service level. This means that the expectations of the user for a composite service do not necessarily mean the sum of each individual expectation, but a combination of both. Such combination depends on both the user and the target individual services.

Based on our top-down approach we just need to start the analysis asking about the objective and subjective requirements of users for such a composite service: What are the user expectations and needs for such a (composite) service? What are their motivations? What is their current context? What would be their reaction? All such questions refer to the QoE domain. On the other hand the Technology and Business Domain aspects should also be considered: What is the business strategy of the operator providing the services? What type of service performance parameters (e.g., QoS) and Service Level Agreements (SLAs) can this operator offer to match QoE requirements of customers? However composition mechanism is not within the scope of this paper, we focus more on how to introduce QoE aspects in service delivery.

Note that the overall system performs two fundamental operations: (i) selecting the optimal services (through a matching mechanism) and (ii) deciding the best delivery method for the user. In both operations QoE parameters are relevant. For example, if the usability factor of a service is high and the user is supposed to have difficulties using it, we can select among those services providing the same functionality with easy-to-use interfaces. This will be a criterion at matching time. For service delivery, if the availability is critical for a user, this maps directly to a QoS parameter (delay, response time, etc.), and we should deliver the service with the corresponding resource allocation to guarantee a certain QoE oriented adaptive SLA.

The QoE evaluation at discovery time is shown in Fig. 2. In this situation the user issues a composite query with his/her requirements and he sends it to the platform front end.

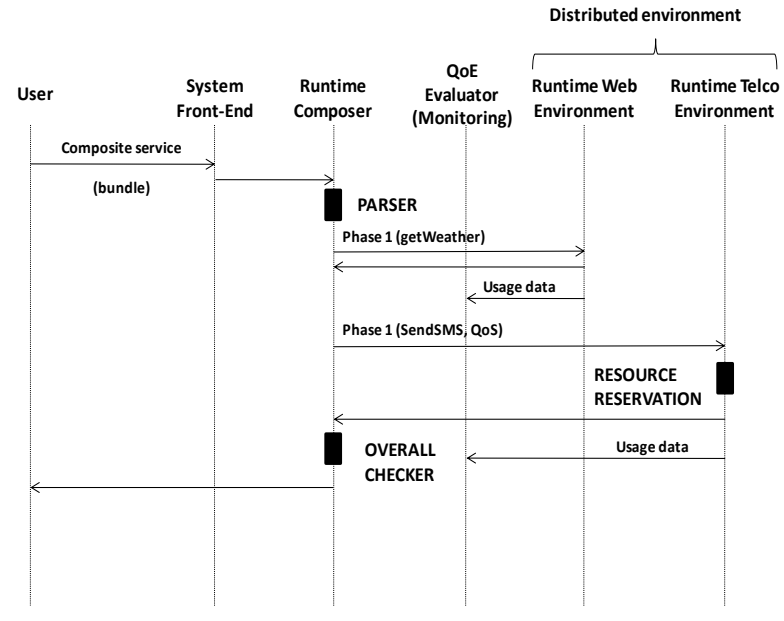

Figure 3. QoE evaluation at runtime

This is passed to the Service Composer, which is in charge of decomposing the query in atomic ones identifying the different services to be provided. For each individual service, it tries to find the optimal service through the Matching Engine. In the first task (getWeather) the Matching Engine searches for a list of matching services in its internal repository, but beforehand it contacts the QoE evaluator to retrieve a list of quality parameters that serve as filtering criteria. Note here that the QoE evaluator replies with parameters taking into account a composite service and the order of each service. This QoE evaluator considers here QoE aspects as described in section III and it includes psychological models, human characteristics and context factors. The Matching Engine answers to the Service Composer with the best service and the same process is done for the second task (sendSMS). Once all tasks are 
done, the Service Composer creates a runtime 'bundle' and sends it to the user. Such bundle describes how this composite service should be later interpreted at runtime.

The QoE evaluation at runtime takes place in a different way, as depicted in Fig. 3. The composite service (special bundle) is sent to the runtime Composer acting as 'proxy' for service execution. It parses the whole service and executes it in atomic parts, targeting both the Web and Telco environment. For the Web world, quality parameters have already been taken into account at discovery time by the Matching Engine. For the Telco environment, special QoS parameters have to be sent so that concrete resources can be allocated at the infrastructure level. However, in both cases (Web and Telco world), feedback to the Monitoring Module is provided in order to assess the overall QoE. The Monitoring Module can be part of the QoE evaluator so that this data is present for future discovery of services.

\section{CONCLUSION}

QoE is a complete assessment of human needs and requirements. In a distributed environment, where multiple services are bundled, each one with diverse QoE requirements poses a big challenge in terms of offering an optimal level of QoE. This paper proposes a QoE interaction model based on multi-disciplinary fields such as human psychology, cognitive factors and service ecosystem to understand the human requirements in a distributed environment. Current work provides a moderate path towards a general framework for assessing QoE requirements for composed services taking into account some subjective and objective QoE parameters and studying the mapping between QoE domain and Technology \& Business domain in order to ensure rich quality of experience.

This paper is an effort to devise means and methodologies to support QoE for service delivery a distributed environment. The paper describes a basic and simplified QoE evaluator based on the QoE interaction model, which focuses on the different time points where mappings takes place, such as service selection and service delivery in order to evaluate the QoE requirements for a demanded service bundle. QoE aware service delivery framework can provide a better service from the user's perspective. Future work will include more use cases on a practical test bed with real users in order to obtain more practical results. We intend to extend QoE interaction model to more formal model and it will also include SLA Management system.

\section{ACKNOWLEDGMENT}

We would like to thank the CELTIC project SERVERY which has partially funded the research presented in this paper. We expect to provide more experimental results by the time the aforementioned project enters an evaluation period.

\section{REFERENCES}

[1] Cisco Whitepaper, "DiffServ-The Scalable End-to-End Quality of Service Model", 2005.

[2] W. Abramowicz, M. Kaczmarek, M. Kowalkiewicz and D. Zyskowski, "Architecture for Service Profiling", Services Computing Workshops, Chicago, USA, Sept. 2006.

[3] S. Richter and A. Bohm, "A location and privacy service enabler for context-aware and location-based services in NGN", Telecommunications Network Strategy and Planning Symposium, New Delhi, India, November 2006

[4] Khalil ur Rehman Laghari,Imen G.ben Yahya,N.Crespi, "Towards Service Delivery based on Customer eXperience ontology: Shift from Service to Experience", $5^{\text {th }}$ IEEE international workshop,MACE2010,Niagara Falls, Canada october 2010.

[5] Hyun Jong Kim,Dong Hyeon Lee,Jong Min Lee,Kyoung Hee Lee,Won Lyu, "The QoE Evaluation Method through the QoS-QoE Correlation Model," Seong Gon Choi 2008 Fourth International Conference on Networked Computing and Advanced Information Management, September 2008.

[6] Peter Reichl, , Sebastian Egger,Raimund Schatz, Alessandro D'Alconzo,"The Logarithmic Nature of QoE and the Role of the Weber-Fechner Law in QoE Assessment," IEEE Communications Society subject matter experts for publication in the IEEE ICC 2010 proceedings.

[7] Markus Fiedler, Blekinge Institute of Technology Tobias Hossfeld and Phuoc Tran-Gia, University of Würzburg,"A Generic Quantitative Relationship between Quality of Experience and Quality of Service." IEEE Network Magazine March/April 2010.

[8] Maslin Masrom, "Technology Acceptance Model and E-learning," 12th International Conference on Education, Sultan Hassanal Bolkiah Institute of Education Universiti Brunei Darussalam 21-24 May 2007

[9] Kay Connelly, "On Developing a Technology Acceptance Model for Pervasive Computing In the Proceedings of Ubiquitous System Evaluation (USE)," a workshop at the Ninth International Conference on Ubiquitous Computing (UBICOMP),September, 2007.

[10] Icek Ajzen 1991. "The Theory of Planned Behavior- Organizational Behavior and Human Decision Processes 50," 179-211(1991). DOI=http://www.courses.umass.edu/psyc661/pdf/tpb.obhdp.pdf

[11] Liu, Y., Feyen, R., \& Tsimhoni, O, "Queuing Network-Model Human Processor (QN-MHP): A computational architecture for multitask performance in human-machine systems." ACM Transactions on Computer-Human Interaction, 13, 37-70.

[12] Bonnie E. John, David E. Kieras 1996, "Using GOMS for user interface design and evaluation: which technique?," $\mathrm{ACM}$ Transactions on Computer-Human Interaction (TOCHI), v.3 n.4, p.287-319, Dec. 1996.

[13] ITU-T Report 2007. "Definition of Quality of Experience (QoE)", International Telecommunication Union, Liaison Statement, Ref.: TD 109rev2 (PLEN/12), Jan. 2007.

[14] Gerd Waloszek,SAP Design Guild "Human performance at the computer_Part 2:Making applications more responsive," SAP User Experience, SAP AG - November 7, 2008.

[15] SERVERY project, http://projects.celtic-initiative.org/servery/ (last visit: October, 11, 2010)

[16] Ndubisi, N.O. (2004), "Factors influencing e-learning adoption intention: Examining the determinant structure of the decomposed theory of planned behaviour constructs," In Proceedings of the 27th Annual Conference of HERDSA, 252-262.

[17] Wanmin Wu, Md.Ahsan Arefin, Raoul Rivas, Klara Nahrstedt, Renata M. Sheppard, Zhenyu Yang "Quality of experience in distributed interactive multimedia environments: toward a theoretical framework.", ACM Multimedia 2009. 